\title{
CIRCULARIDAD MIGRATORIA ENTRE ECUADOR Y ESPAÑA. TRANSFORMACIÓN EDUCATIVA Y ESTRATEGIAS DE MOVILIDAD
}

\section{MIGRATORY CIRCULARITY BETWEEN ECUADOR AND SPAIN. EDUCATIONAL TRANSFORMATION AND MOBILITY STRATEGIES}

Cristina Vega Solís, Carmen Gómez Martín y Ahmed CorreA*

\begin{abstract}
Resumen: El patrón migratorio entre Ecuador y España ha experimentado transformaciones desde el inicio de la crisis de 2008. A la ralentización de los flujos hacia España y el incremento de retornados ecuatorianos, se suma hoy la migración de españoles cualificados hacia este país para insertarse en educación superior, así como la salida de jóvenes ecuatorianos para adquirir títulos de posgrado en España y, en general, en Europa. El contexto de crisis y desempleo en educación en España, así como las politicas de reforma educativa que se están llevando a cabo en Ecuador se articulan en la conformación de esta circulación entre ambos países. A través de una metodología cuantitativa y cualitativa multisituada, basada en una encuesta y en entrevistas en profundidad, el presente artículo estima el volumen de estos desplazamientos y explora las motivaciones, condiciones y estrategias que los están influenciando.
\end{abstract}

Cristina Vega es profesora-investigadora. Coordinadora de la Maestría de Género y Desarrollo del Departamento de Sociología y Estudios de Género. Facultad Latinoamericana de Ciencias Sociales (Flacso-Ecuador). Carmen Gómez Martín es profesora-investigadora. Departamento de Sociología y Estudios de Género. (Flacso-Ecuador) y Ahmed Correa Álvarez es investigador asociado. Departamento de Sociología y Estudios de Género.(Flacso-Ecuador). 
Palabras clave: migración cualificada, circularidad migratoria, educación superior, Ecuador, España.

Abstract: The migration pattern between Ecuador and Spain has undergone changes since the start of the 2008 crisis. A slowdown in inflows to Spain and increasing Ecuadorian returnees, today add the Spanish skilled migration to this country in entering higher education as well as the exit of young Ecuadorians to acquire fourth level's degree in Spain, or Europe in general. The context of crisis and unemployment in education in Spain and education reform policies being carried out in Ecuador is articulated in shaping this circularity between the two countries. Through a quantitative and qualitative multi-situated methodology based on a poll and in-depth interviews, this paper estimates the volume of these displacements and explores the motivations, conditions and strategies that are influencing.

Keywords: skilled migration, circular migration, higher education, Ecuador, Spain.

\section{INTRODUCCIÓN}

La crisis en España y las reformas institucionales en Ecuador están modificando el patrón de movilidad entre ambos países. Por más de una década, los ecuatorianos han migrado a España en busca de trabajo en sectores descualificados, ocupando un lugar desaventajado en un mercado laboral fuertemente estratificado. En la actualidad, se detecta una creciente salida de jóvenes titulados en sentido inverso con propósitos laborales. Si bien la expulsión de españoles cualificados no es un fenómeno nuevo, el sentido NorteSur, particularmente hacia América Latina, constituye una novedad que amerita un estudio más detallado.

La migración cualificada entre España y Ecuador no se limita a los españoles. Gracias al programa de becas del gobierno ecuatoriano, cada vez más población de este país encuentra las condiciones propicias para desplazarse por motivos formativos. La reforma de la educación superior, con exigencias de títulos de posgrado, y la asimetría en el acceso y valor de los mismos hacen que un número creciente de ecuatorianos completen su formación en el 
Norte. Existiría, por lo tanto, un patrón migratorio interdependiente vinculado a la inserción laboral y formativa.

Tras un primer apartado metodológico, el texto aborda estas movilidades desde la perspectiva transnacional y la teoría de la circularidad migratoria. Posteriormente, se ofrece una aproximación al contexto de crisis que condiciona el desplazamiento de jóvenes cualificados españoles y el entorno de oportunidades y reformas institucionales en Ecuador que influyen en el desplazamiento formativo de ecuatorianos y la recepción de españoles. Finalmente, se abordan los principales hallazgos de la investigación: una estimación del volumen de la circulación de población en educación superior entre ambos países, un análisis de las motivaciones y dinámicas de movilidad de estas poblaciones, así como una reflexión sobre las condiciones y modos de inserción en los contextos de destino.

\section{METODOLOGÍA}

Las informaciones contenidas en este artículo corresponden a dos investigaciones realizadas en $2014^{1}$, en las que se combinaron enfoques cualitativos y cuantitativos. El levantamiento de información y datos se apoyó en varias fuentes: a) datos de instituciones ecuatorianas y españolas ${ }^{2}$; b) seis entrevistas en profundidad a directivos y funcionarios de instituciones públicas ecuatorianas y españolas; c) una encuesta aplicada a 184 españoles profesores e investigadores en universidades ecuatorianas.

1 Procesos de inserción socio-laboral de la migración calificada en Ecuador, financiado por la Dirección General de Empleo de España, y Circularidad migratoria entre Ecuador y España. Transformación educativa y estrategias de movilidad, financiado por Flacso-Ecuador. El equipo de investigación está integrado además por Rocío Pérez, Lucía Pérez y Silvina Monteros.

2 Ministerio de Relaciones Exteriores y Movilidad Humana (MREMH), Secretaría Nacional de Educación Superior, Ciencias, Tecnología e Innovación (SENESCYT), SRI, Instituto Ecuatoriano de Crédito Educativo (IECE), Instituto Ecuatoriano de Seguridad Social (IESS), Instituto Nacional de Estadística y Censos (INEC), Ministerio de relaciones laborales (MRL), Ministerio de Educación Ecuatoriano, Ministerio de Salud Ecuatoriano, Consulados de España en Quito y Guayaquil. 
TABLA I

POBLACIÓN DE LA ENCUESTA POR SEXO Y GRUPO DE EDAD

\begin{tabular}{|c|c|c|c|c|}
\hline \multicolumn{5}{|c|}{ SEXO } \\
\hline & 121 & \multicolumn{2}{c|}{ Mujer } & Total \\
\hline Casos & \multicolumn{6}{|c|}{ GRUPOS DE EDAD } \\
\hline \multicolumn{5}{|c|}{} \\
\hline \multirow{2}{*}{ Casos } & $26-34$ años & $35-44$ años & $\begin{array}{c}45-54 \\
\text { años }\end{array}$ & 55 y más años \\
\hline & 62 & 60 & 38 & 24 \\
\hline
\end{tabular}

Fuente: encuesta.

d) 116 entrevistas semi-estructuradas distribuidas en cinco grupos: 81 a docentes e investigadores españoles en universidades ecuatorianas -incluyendo los investigadores del programa Prometeo- $-\mathrm{y}$ estudiantes; 35 entrevistas a ecuatorianos, 18 a estudiantes o pro-fesores retornados, ubicados en universidades ecuatorianas y 17 repartidas entre estudiantes de posgrado en universidades españolas y jóvenes ecuatorianos residentes en España que aspiran a integrarse en universidades españolas o ecuatorianas.

Con el fin de dar cuenta de la interdependencia en las movilidades desarrollamos una metodología «multi-situada» (Gil, 2006) entre España y Ecuador. En España, el trabajo de campo se realizó en Madrid, Murcia y Granada. En Ecuador, la investigación tuvo lugar en las ciudades de Quito, Cuenca y Loja.

\section{APROXIMACIÓN TEÓRICA: CIRCULARIDAD MIGRATORIA, MIGRACIÓN CUALIFICADA Y MOVILIDADES INTERDEPEN- DIENTES}

El concepto de circularidad migratoria proporciona, en un marco de aproximación transnacional, una herramienta útil para pensar la movilidad de personas cualificadas en el ámbito de la educación superior. Arab (2008) explica cómo la territorialidad en la migración, especialmente a partir de la década de 1990, se dirime no en el polo origen y destino, sino en la circulación. Así, más que «migrar», muchas 
personas «circulan» entre espacios, sin que la idea de un arribo definitivo, permanente o estable se sitúe en el centro de su proyecto de desplazamiento (Hily y Ma Mung, 2003). Los movimientos incesantes entre distintos lugares forman parte de este concepto de circulación.

Mientras la integración había sido clave para pensar la migración de los sesenta y setenta en el contexto europeo, en la actualidad, las interrogantes se dirigen a entender los encuentros, negociaciones e interacciones entre espacios. A la atención hacia los lugares de estancia, se añade la que se dirige hacia los espacios de tránsito, que se conectan de modos diversos y multiescalares (internacional, regional, nacional y local), y que ya no se pliegan a un único orden de integración nacional normativa en las sociedades de «recepción». En efecto, las relaciones económicas, sociales, políticas y culturales entre espacios, personas y mercancías no pueden ser solamente descritas por las dinámicas bidireccionales push/pull.

Un territorio circulatorio es, según Tarrius (2000), un espacio cuya territorialización pasa justamente por el movimiento. Éste adquiere sentido social, y no se reduce a dos localizaciones (origen y destino), sino que se produce en etapas y tránsitos que pueden tener un lugar central en la experiencia. Independientemente de si los migrantes circulan o no y del modo en que lo hagan, el vivir transnacional se afirma en los múltiples campos en los que se dirime la vida migrante (Glick Schiller, Basch y Szatón-Blanc, 1999; Guarnizo, Portes y Haller, 2003).

Los estudios sobre migración cualificada ponen de relieve el dinamismo de la circulación y los modos de ser transnacionales ${ }^{3}$. Al igual que otros mercados globales, éste ha experimentado un claro proceso de integración mundial. Esto se deja ver de forma especial en la movilidad en educación superior (formación, docencia, investigación, redes) estimulando las reformas educativas intercambios, transferencias de conocimientos y programas de movilidad entre instituciones académicas como parte de lo exigido a los profesionales de este sector.

Así, se espera de quienes aspiran a insertarse en este mercado que quieran y puedan moverse, haciendo carreras en las que el dinamismo representa un valor añadido. Quienes se movilizan despliegan un saber-hacer-en la movilidad basado en conocimientos,

3 Las tasas de emigración mundiales muestran que la mayoría de los migrantes son profesionales cualificados: poco cualificados $(0,9 \%)$, cualificación media $(1,6 \%)$ y altamente cualificados (5,5\%) (Docquier y Rapoport, 2006). 
redes, contactos y ayudas como parte del desarrollo de una carrera profesional (Tarrius, 2000: 51). En el presente estudio, la movilidad está condicionada por la economía mundial del conocimiento, en la que los países cuentan con capacidades formativas desigualmente valoradas y mercados de trabajo con diferentes oportunidades. Es importante advertir que ambas cosas no siempre van de la mano. Actualmente, los títulos europeos siguen siendo prestigiosos en América Latina, si bien pocas son las oportunidades de mantenerse en el mercado de educación superior en Europa una vez finalizada la formación. Esta geografía diferencial, propia de la globalización, hace que las colectividades nacionales circulen «en búsqueda» de recursos o trabajos, y que estas movilidades aparezcan interconectadas.

Tanto la perspectiva del «brain drain $»^{4}$, como la del «brain circulation» son, a nuestro juicio, limitadas. La primera, a pesar de contemplar la geografía desigual del conocimiento, presenta un marco excesivamente dicotómico y unidireccional. El drenaje responde a las relaciones desiguales inscritas en el sistema económico capitalista basado en el beneficio de una parte en detrimento de otras. El diferencial de preferencia (ingreso, apoyo logístico y reconocimiento profesional), junto a la situación política y el estado del sistema universitario (Oteiza, 1971), captaría las opciones de estos migrantes. Esto tiene fuertes implicaciones al producir encajes dinámicos entre unos países con «déficits educativos» y otros donde los cualificados no pueden insertarse en el mercado laboral, sufriendo constantes procesos de descualificación (Sevilla, 2010).

La segunda, por contra, capta el dinamismo en los desplazamientos temporales y pendulares. La circularidad se refiere a múltiples salidas y retornos, con frecuencia animadas por políticas de atracción (temporal) de talentos y otras que comprometen el retorno de quienes se forman fuera. El problema aquí es, más bien, que no siempre se hace manifiesto que los movimientos no son un mero flujo nómada libre y autodeterminado (Sheller y Urry, 2014), sino que dependen de una geografía desigual, en este caso, del conocimiento y la educación.

En esta investigación, el concepto de circularidad recoge también otra característica importante: la interdependencia de los

\footnotetext{
4 Docquier y Rapoport (2006: 2) definen el «braindrain» como la «transferencia internacional de recursos en la forma de capital humano que ocurre especialmente como migración de individuos altamente cualificados desde países en vía de desarrollo a países desarrollado».
} 
desplazamientos ${ }^{5}$. La reforma de la educación superior en América Latina ha implicado tanto la salida de unos - quienes buscan formación- hacia Europa y Estados Unidos, como la llegada de otros - quienes ya tienen dicha formación y buscan trabajo- desde Europa. En nuestra aproximación, estas dos movilidades están conectadas. Puede que muchos migrantes cualificados se desplacen a un tercer país; indudablemente, su movilidad afectará a las expectativas laborales de «los que se fueron a volver» y de los hoy que llegan. En sintonía con recientes enfoques sobre las migraciones, y aunque aquí no nos detendremos en ello, es preciso señalar que las motivaciones profesionales se articulan de forma compleja con otras de carácter familiar, afectivo, cultural y político en la definición del proyecto migratorio (Sanz Abad y García-Moreno, 2012)

\section{CONTEXTUALIZACIÓN: PATRONES MIGRATORIOS, EDUCACIÓN SUPERIOR Y MERCADO DE TRABAJO}

Se tiende a pensar que la crisis del 2008 ha modificado los patrones migratorios en España, sobre todo los relativos a América Latina y, especialmente, a Ecuador, volviéndose a dinámicas de emigración ${ }^{6}$. Esta concepción es, sin embargo, simplista. La crisis económica no rompe con los flujos migratorios hacia España, simplemente los complejiza (Cachón, 2012).

En el caso de Ecuador, las migraciones hacia los Estados Unidos cambian con la crisis económica ecuatoriana de finales de los noventa (Jokisch y Kile, 2005; Pedone, 2005; Ramírez y Ramírez, 2005). España e Italia se transforman en los principales centros de atracción ${ }^{7}$ debido al boom inmobiliario y a la existencia de mercados laborales segmentados que requieren de mano de obra migrante:

5 Otras teorizaciones como la de cadenas migratorias para el cuidado captan esta misma idea de interconexión entre movilidades (Hochschild, 2001). Si bien la idea de cadena tiene algunas limitaciones, lo interesante es que ilustra la existencia de nodos interdependientes.

6 Entre 2013 y 2012 la inmigración descendió un 4,3\% y la emigración experimentó un incremento del 22,7\% (INE, 2014).

7 Según el censo de 2001, el 49,40\% de la población que salió entre 1996 y 2001 se dirigió a España; el 26,70\% a Estados Unidos y el 9,90\% a Italia. En España los ecuatorianos pasaron de 13.000 empadronados en 1998, a cerca de 500.000 en el 2005 (Herrera, 2007: 193-194). 
hombres en agricultura y construcción y mujeres en cuidado y trabajo doméstico (Herrera, 2007).

España, por su parte, se transforma en lugar de inmigración a partir de los años ochenta. En esta década y particularmente en la siguiente, la emigración se vincula a una población con elevados niveles de cualificación (Alaminos y Sancreú, 2010), que sale al exterior para trabajar o estudiar postgrados. Dominan aquí dos patrones de movilidad: el europeo, relacionado con las oportunidades del espacio Schengen ${ }^{8}$ y el latinoamericano, marcado por la cooperación internacional y las grandes empresas transnacionales (Sallé Alonso, 2009).

La crisis complejiza estos patrones migratorios a través del aumento de retornos de ecuatorianos ${ }^{9}$ y consolidación de flujos de poblaciones cualificadas, tanto de españoles para trabajar en América Latina, como de ecuatorianos para titularse en Europa.

\section{España: condicionantes laborales y desconexión entre la educación superior y el mercado de trabajo}

Más allá de los efectos que provoca la crisis en las estructuras productivas y laborales de España ${ }^{10}$, han sido las medidas de «austeridad» las que han terminado por agudizar y dilatar la crisis y sus efectos en términos de bienestar (Navarro et al., 2011, López y Rodríguez, 2010).

Es necesario aclarar, sin embargo, que las dinámicas de movilidad actuales son anteriores; están ligadas a la crisis económica española de principios de los noventa, que dejó tasas de desempleo del 24\% (1993). La reforma laboral de 1994 pretendió atajar los problemas impulsando el empleo temporal ${ }^{11}$, dejando el camino libre para la

8 Según el INE, entre 2002 y 2007, un total de 77.293 españoles emigraron a otros países europeos. Reino Unido (17.414), Francia (13.713), Alemania (10.243) e Italia (4.468).

9 Regreso que, como atestiguan trabajos sobre el retorno en Ecuador (Moncayo, 2011; Herrera et al., 2012; Vega 2014), no tiene por qué transformarse en destino final.

10 La Encuesta de Población Activa del segundo trimestre del 2014 señala un total de 5.622.860 parados en España.

11 Según Polavieja (2006), desde 1990 el mercado español presenta las tasas de empleo temporal más altas de toda la OCDE. Según la Encuesta de Población 
precarización (juvenil) del mismo (Polavieja, 2006; Gómez y de la Calle, 1995). En este sentido, las reformas laborales de los gobiernos de Zapatero (2010) y Rajoy (2012) han degradado progresivamente los derechos laborales, apuntalando la «flexibilización» como forma para combatir el desempleo.

Esto ha provocado la reactivación de los flujos migratorios de cualificados que sufren la desconexión entre el sistema educativo, dirigido hacia los estudios universitarios, y las demandas reales de la estructura productiva (Polavieja, 2006; Gómez y de la Calle, 1995). En definitiva, encontramos por un lado una mano de obra que acaba transformándose en capital inutilizado o subutilizado (Prats, 2005: 212), mientras que, por otro lado, el sistema educativo — debilitado por los recortes ${ }^{12}$ - también genera dinámicas migratorias debido a las desigualdades de acceso debidas al encarecimiento de las tasas universitarias $^{13}$ que afectan a las clases medias empobrecidas y los hijos de poblaciones migrantes.

\section{La Reforma educativa ecuatoriana y la movilidad de cualificados}

Ecuador vive desde 2006 una profunda reestructuración del Estado encabezada por Alianza País, cuyas líneas directrices se encuentran en los llamados «Planes del Buen Vivir» 2009-2013 y 2013-2017, en los que la cuestión educativa ha tenido un papel prioritario de inversión ${ }^{14}$.

La Ley Orgánica de Educación Superior (LOES) de 2010 fija las condiciones que deben cumplir las Universidades en ese proceso, elevando el estándar de calificación de las universidades,

Activa española desde el 2007 los contratos temporales crecieron un 48.2\%. De los contratos registrados en 2014 (14.241) solo 1.433 son indefinidos, frente a un total de 12.808 contratos temporales (INE, 2014: 244).

12 La reducción del gasto público en educación alcanza el 37\% entre 2010 y 2012. El Real Decreto-Ley 14/2012 de 20 de abril, y el Real Decreto 1000/2012 de 29 de junio, son significativos de estos recortes.

13 Las subidas de tasas académicas llegaron hasta los 540 euros en 2012. Ver: http://sociedad.elpais.com/sociedad/2014/06/23/actualidad/1403540149_298764. html

14 En 2013 se crean 4 universidades emblemáticas: Universidad Nacional de Educación, Universidad Regional Amazónica, Universidad de las Artes y Universidad de Investigación de Tecnología Experimental. 
priorizando la contratación de personal con títulos de Doctorado, etc. Para paliar la escasez de doctores, el gobierno ha implementado diversos programas.

En primer lugar, la captación de personal extranjero o ecuatoriano cualificado residente en el exterior a través de llamamientos propios de las universidades o de programas de excelencia para promover procesos de formación y cualificación de profesionales nacionales. Es el caso del Programa Prometeo ${ }^{15}$ y del megraproyecto de desarrollo científico y tecnológico Ciudad del Conocimiento Yachay ${ }^{16}$. Según Héctor Rodríguez, gerente general de la Empresa Pública Yachay, la captación de personal cualificado extranjero y ecuatoriano procedente del exterior se justifica porque:

(...) Según la línea base 2010, tenías apenas 600 investigadores con el grado de $\mathrm{PhD}$, que se dedicaban a actividades de desarrollo en el país a tiempo completo... 600, siendo prácticamente doce millones de habitantes. (...). La política de becas marcaba una distensión en el espacio y en el tiempo para poder lograr esa masa crítica, entonces ante la imposibilidad de contar inmediatamente tienes que importar. Yachay consciente de esos tiempos se apura en la repatriación de cerebros, mostrando ventajas comparativas y ventajas competitivas respecto, en el plano muchas veces al tema del nacionalismo etc., pero también a la, ventaja material que tienes acá. Digamos salarios competitivos, por otro lado cercanía cultural y el tema, digamos en tema de poder iniciar algo (H. Rodríguez. Quito, junio 2014).

En segundo lugar, se fomenta el reforzamiento de la formación de ecuatorianos en el exterior, promoviendo los retornos de personas becadas para evitar así la «fuga de cerebros» (Ramírez, 2010: 22). Aquí nos encontramos los programas de becas de la SENESCYT, creados para brindar ayudas económicas a ecuatorianos que quieran desarrollar cursos de formación de grado y posgrado en universidades extranjeras, o el sistema de "Crédito Educativo y Becas» del Instituto Ecuatoriano de Créditos Educativos (IECE).

\footnotetext{
15 http://www.prometeo.com.ec/

16 http://www.yachay.gob.ec/
} 


\section{HALLAZGOS: DIMENSIONANDO LAS MOVILIDADES CUALIFICADAS EN EDUCACIÓN SUPERIOR: TRAYECTORIAS Y SIGNIFICADOS}

\section{Exageraciones y subregistros en la circulación de cualificados españoles y ecuatorianos. ¿Qué está en juego?}

Existe un baile de cifras dependiendo de las fuentes sobre la población cualificada circulando entre ambos espacios. El uso intencional que se hace de la ausencia de datos concluyentes muestra que detrás del tratamiento de la movilidad humana existen finalidades políticas concretas e intereses en disputa. Los medios de comunicación se hacen eco de esta situación, pero también la academia entra de lleno en el debate. Así por ejemplo, dependiendo de las fuentes estadísticas utilizadas y de la lectura de los datos, encontramos autores que rebajan el tono de alarma con respecto a la salida de españoles y otros que critican la interpretación de las cifras a la baja ${ }^{17}$.

La disparidad en la estimación se produce en general respecto a los que se desplazan hacia América Latina. En este continente reside el mayor número de españoles en el exterior, aunque su cuantificación a menudo incluye a los retornados latinos nacionalizados, a sus hijos, nacidos en España, y a los naturalizados bajo la Ley de Memoria Histórica. Ecuador es el país latinoamericano con mayor incremento en la llegada de nacionales españoles ${ }^{18}$, pero estas cifras están además conectadas con procesos de retorno de migrantes ecuatorianos naturalizados y sus hijos (González, 2013).

En términos generales, el debate en torno a la migración de los españoles de origen se ha polarizado tanto en España como en Ecuador. Para el gobierno español se trata de «aventureros ${ }^{19}$,

17 González (2013), basándose en el Padrón de Españoles Residiendo en el Extranjero (PERE), mostraba que el número de españoles residentes en el exterior entre 2009 y 2013 era de apenas 40.000 personas y, de éstas 7.372 corresponden a españoles nacidos en España. Por su parte, González-Ferrer (2013) critica las limitaciones de los datos del PERE y sitúa las salidas entre 2008 y 2012 en 700.000 personas.

18 Según el INE (2014), Ecuador se convirtió en 2013 en el tercer país de recepción de españoles a nivel mundial, después del Reino Unido y Francia.

19 Para Marina del Corral, Secretaria General de Inmigración y Emigración, la emigración de jóvenes no se debe sólo a la crisis sino a otros factores positivos, 
mientras que para quienes critican esta visión nos hallamos ante un «exilio laboral $»^{20}$. En Ecuador, por contra, las interpretaciones oscilan, particularmente en lo que se refiere a la educación superior, entre contemplarlos como «sabios» ${ }^{21}$ cuyo talento humano dinamiza el sistema educativo o "colonizadores» que desplazan a los académicos locales y regionales. Por otra parte, las visiones sobre aquellos que se desplazan por motivos formativos se utilizan como emblema de las potencialidades del nuevo sistema educativo en el desarrollo del país.

De acuerdo al Informe de Acciones 2013 de la Embajada española en Ecuador, el total de personas de nacionalidad española registradas en este país (incluyendo residentes y no residentes, así como españoles de origen y nacionalizados) era de 38.132 a diciembre de 2013.

TABLA II

NACIONALES ESPAÑOLES REGISTRADOS POR AÑO

\begin{tabular}{|c|c|c|c|c|}
\hline Año & 2010 & 2011 & 2012 & \\
\hline Registrados & 14.527 & 19.554 & 28.001 & 38.132 \\
\hline
\end{tabular}

Fuente: Consulado de España en Ecuador.

La información desagregada nos dice que hasta finales de 2013 había un total de 25.909 personas retornadas o hijos de éstas, y que solamente 12.202 eran autóctonos españoles. Teniendo en cuenta que éstos últimos no suelen inscribirse en los consulados, resulta difícil leer en estos datos cuál ha sido el incremento real entre 2010 y 2014, pues la cifra responde al acumulado desde que se tiene registros. No obstante, tomando los datos de visas a españoles que nos aporta el Ministerio de Relaciones Exteriores y Movilidad Humana de Ecuador (MREMH) entre 2010 y 2014 (7.650) y los saldos migratorios desde 2012 a 2014 (8.100), podemos

entre ellos, «el espíritu aventurero de la juventud». http://politica.elpais.com/ politica/2012/11/30/actualidad/1354286966_753467.html

20 http://www.nonosvamosnosechan.net/p/exilio-labroral.html

21 Denominación incluida en el programa Prometeo. 
cifrar aproximadamente las llegadas de españoles autóctonos en los últimos 5 años entre 7.000 y 7.500. El incremento de llegadas puede advertirse en las siguientes tablas:

TABLA III

VISAS A CIUDADANOS ESPAÑOLES ENTRE 2010 Y 2014

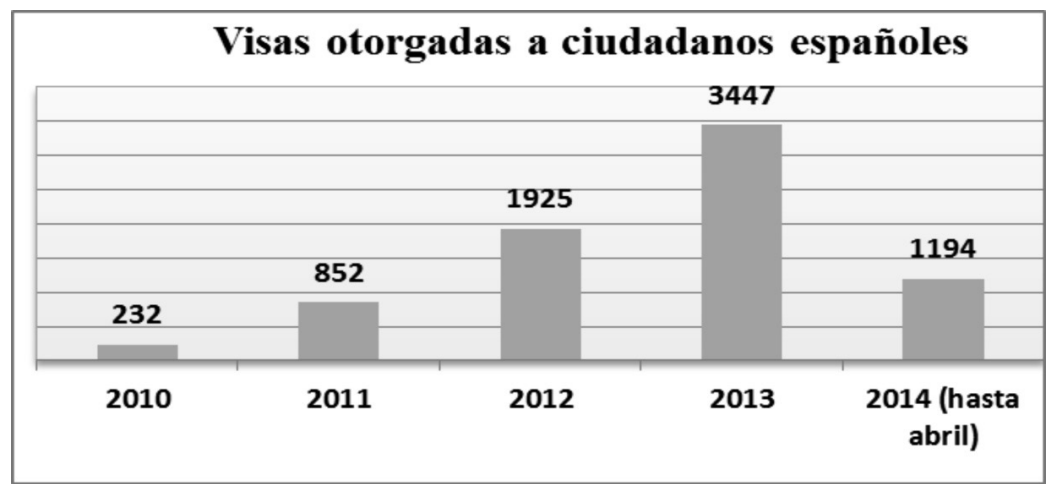

Fuente: MREMH.

TABLA IV

SALDOS MIGRATORIOS EN ECUADOR DE POBLACIÓN CON NACIONALIDAD ESPAÑOLA ${ }^{22}$

\begin{tabular}{|l|l|l|l|}
\hline \multicolumn{1}{|c|}{ Años } & Ingresos & Salidas & Saldos \\
\hline 2012 & 65.765 & 61.813 & 3.952 \\
\hline 2013 & 65.159 & 61.266 & 3.893 \\
\hline Abril 2014 & 18.006 & 17.751 & 255 \\
\hline Total & 148.930 & 140.830 & 8.100 \\
\hline
\end{tabular}

Fuente: MREMH.

22 En esta información no consta la desagregación de ecuatorianos naturalizados. 
Un número importante de estos españoles están empleados en puestos cualificados, incluyendo la educación superior. Según nuestras estimaciones, en el ámbito universitario podrían situarse entre los 500 y los 600, entre docentes, investigadores prometeos, otros investigadores y estudiantes ${ }^{23}$. Según la encuesta realizada en 2014 se trata mayoritariamente de varones en una proporción de 2 a 1 (121 hombres/63 mujeres), de entre 26 y 44 años y solteros, aunque el 42,5\% afirma vivir en Ecuador con su pareja (mayoritariamente española y el $15 \%$ dice tener pareja ecuatoriana) y el $15,5 \%$ tener hijos ${ }^{24}$.

El gobierno y algunos medios de comunicación ecuatorianos subrayan la capacidad de atracción del país, ya sea para retornados como para extranjeros ${ }^{25}$. Los llamamientos hechos incluso desde España $^{26}$ son una clara muestra del interés del Estado ecuatoriano y de instituciones como la SENESCYT por visibilizar y acoger a estos profesionales.

Esta publicidad ha generado rechazo entre algunos docentes ecuatorianos, que conectan la llegada de profesionales extranjeros, especialmente de españoles, con los privilegios que se desprenden de su incorporación a puestos de prestigio. Si bien las cifras de profesores e investigadores españoles es pequeña ( $0.3 \%)$, su notable presencia en programas como Prometeo (33\%) ha condicionado la percepción de masividad. Entendemos, por consiguiente, que la visibilidad de estos profesionales no se encuentra en una abultada inserción en detrimento de los nacionales, sino en los puestos de poder y decisión que ocupan, en las exorbitantes remuneraciones ofrecidas de programas como Prometeo y, en general, en las excelen-

23 La estimación se basa en el número de profesores registrados hasta 2012 en el Sistema Nacional de Información de la Educación Superior del Ecuador (SNIESE), el número de Prometeos registrados hasta mayo de 2014, el número de universitarios españoles recogido por SENESCYT (un total de 68, 53 en maestría y 15 en doctorado) y el número de profesionales vinculados a Yachay. Este cómputo no incluye a investigadores independientes, consultores, etc.

24 En los siguientes apartados, los datos corresponden a la encuesta realizada en el marco de este proyecto.

25 Ver la entrevista realizada en diciembre de 2014 a Rafael Correa:

http://www.ecuavisa.com/articulo/noticias/actualidad/91746-actualmentehay-30000-espanoles-ecuador-informo-correa

26 Véase como ejemplo el llamamiento de la subsecretaría de Desarrollo Profesional Educativo de Ecuador, Miriam Aguirre Montero, en Madrid en 2013.

http://sociedad.elpais.com/sociedad/2013/07/22/actualidad/ 1374496004_364310.html 
tes condiciones que experimentan respecto a los académicos nacionales ${ }^{27}$ en un contexto de reformas que incluye exigencias que con frecuencia están fuera del alcance de los titulados locales.

El aumento de ecuatorianos en circulación también ha sido notable. Algunos son retornados atraídos por los programas del gobierno, pero sobre todo se trata de jóvenes, en su mayoría becados, en búsqueda de mayor cualificación. Hasta abril del 2014, había 608 becarios ecuatorianos cursando programas académicos en 46 universidades de España, y otros 270 próximos a efectivizar su beca. Además, 79 de las postulaciones se presentaron directamente desde España por hijos de migrantes ecuatorianos. El IECE, por su parte, entregó entre 2011 y el 2013 un total de $\$ 33.742 .220$,68 en créditos educativos a 3.456 personas que estudiaban en España. Existe además un amplio consenso sobre lo positivas que son estas becas para moverse, siempre y cuando los «retornos» alienten el desarrollo nacional. Finalmente, la captación de retornados cualificados representa un legítimo argumento para el gobierno en el modelo de desarrollo nacional, sin embargo, hasta el momento los programas no arrojan resultados particularmente exitosos en el ámbito educativo.

\section{Motivaciones y trayectorias en la movilidad}

En el colectivo de los españoles en educación superior es preciso establecer una distinción entre dos grupos con motivaciones, condiciones y trayectorias divergentes: el de aquellos en movilidad temporal con vínculos laborales estables en España, en su mayoría investigadores del programa Prometeo, y aquellos con una estrategia migratoria insertos en la academia ecuatoriana. Su desplazamiento depende de sus condiciones de vida y de trabajo y de sus perspectivas a medio plazo.

En el segundo grupo es clara la referencia a la crisis como motivación fundamental para desplazarse. Según los datos de la

$27 \quad$ En mayo de 2014 el número de prometeos españoles alcanzaba las 202 personas, lo que representa el 33\% del total del programa, seguidos por ecuatorianos (12.6\%) y venezolanos (10.4\%). El salario puede llegar a los 6.000 usd (4.500 euros). Además, incluye cinco meses de alquiler (500 usd), seguro médico, un fondo para comprar libros (2.000 usd) y para viajes académicos (2.500 usd) (SENESCYT). 
encuesta, el 39\% dice haber salido por carecer de trabajo, el 45,7\% por la inestabilidad laboral y el $33,7 \%$ por los bajos salarios. Para los primeros, lógicamente, la crisis se revela como "poco importante»; el del 46,2\% mantiene vínculos laborales en origen y el 57,5\% tiene «obligación» contractual de regresar.

Las trayectorias de estos colectivos también son diversas. Su visión sobre el desplazamiento oscila entre una lógica de estancia temporal más o menos prolongada y el tránsito y movilidad a corto y medio plazo hacia otros destinos. Esto dependerá de sus condiciones de vida y trabajo, así como de sus perspectivas profesionales, afectivas y familiares.

También se dan importantes diferencias entre quienes llegan mediante un concurso u ofrecimiento $(57,6 \%)$ y quienes optan al mismo una vez en el país $(42,4 \%)$. Si lo comparamos con los datos anteriores vemos que aproximadamente un $10 \%$ de los investigadores Prometeo apostaría por una estrategia de tanteo de oportunidades para quedarse como parte de una iniciativa migratoria laboral. La expectativa de movilidad queda patente en este segmento.

Yo trabajaba en algunos proyectos de investigación en el departamento (España)... pero cada vez había menos dinero para investigación, y los proyectos son más escasos... tuve mucha suerte. Yo no era profesor en la universidad, soy investigador... Eché los papeles para el Prometeo y me cogieron [...] He pedido el máximo tiempo, un año, y voy a solicitar el segundo año [...] Ahora en abril vuelvo a España a casarme con mi novia y a volver juntos para solicitar el visado de amparo. Ella es abogada, pero trabaja en una tienda, quizás pueda trabajar aquí (Ecuador), de lo suyo [...] pero yo quiero volver a España... me doy un plazo, como mucho, de cuatro años más para regresar. No sé si estaré los cuatro años en Ecuador, quizás me vaya a Europa, pero en cuatro años, me vuelvo a España. (Luis, investigador prometeo, Cuenca, 2014).

Si bien, en una primera fase, parece que las llegadas eran más planificadas, en los últimos años arriban más españoles a «buscarse la vida $»^{28}$. No olvidemos que sólo el 12,5\% de los académicos migrantes

28 Según la encuesta realizada, muchos llegan como turistas (31\%) y más tarde tramitan otro tipo de visado, hecho que indica una migración no planificada. Tanto los trámites en el MRL como en el SENESCYT muestran un incremento en la emisión de autorizaciones laborales y en el registro de títulos (1.248 títulos registrados; 400 de posgrado y 673 de grado). 
tienen nombramiento, mientras que el $28 \%$ están en relación de dependencia y el 18,5\% carecen de dicha relación contractual. Así pues, el grupo de quienes rastrean posibilidades crece y registra una fuerte movilidad, tanto interna como internacional. Se advierte además, como muestra el siguiente testimonio, que esta misma situación impulsa la llegada de estudiantes para cursar en Ecuador.

... la principal razón es porque no tengo mucho que hacer allí actualmente. O sea, después de la experiencia que tuve laboral allí, en tema de investigación, pues viví una realidad bastante cruda, donde vi gente con doctorados y con la experiencia muchísimo más grande que la mía estaban siendo despedidos, ¿no? (...), porque hay gente que lleva un año, dos años de inactividad, en todos los sentidos. Entonces, pues eso también me da miedo, y ésa es una de las razones por las que me vine así, sin pensarlo. Terminé mi licenciatura allá y planeé las maestrías porque..., porque no quería llegar a una situación de vacío existencial. (Antonio, estudiante, Quito, 2014).

Las diferencias de género merecen ser señaladas, ya que como indica la SENESCYT, la presencia masculina es abrumadora tanto entre los investigadores Prometeo (77\% frente a $23 \%$ ), como entre los contratados (61\% y 39\%). Esto, además de poner de manifiesto la propia estratificación de la academia española, revela el impulso de las académicas a la hora de buscar mejores opciones en el exterior.

Otro dato relevante se refiere a los países de residencia anterior de estos profesionales. En la encuesta se recogieron hasta 46 países ${ }^{29}$ de estancia en los 10 años previos a la llegada a Ecuador. El promedio de residencia en estos países es de casi 9 meses y 12 meses en Ecuador. Se trata de profesionales en tránsitos más o menos prolongados, lo cual evidencia las condiciones de este colectivo, pero también que la expulsión de cualificados en España es previa al estallido de la crisis. Las expectativas respecto al futuro también ponen de manifiesto que esta población, si bien afirma desear volver a España a medio plazo, está dispuesta a desplazarse a terceros países. Sólo 6,5\% desea permanecer en Ecuador.

Otro hallazgo es la concentración y movilidad interna de este colectivo. En no pocos casos, profesionales que llegan a universidades en ciudades más pequeñas, son posteriormente contactados y contratados

29 Dentro de la UE, los más comunes son Reino Unido y Francia y en el continente americano aluden a México, Brasil, Estados Unidos y Argentina. 
por universidades prestigiosas de ciudades como Quito y Cuenca, lo cual implica una pérdida para las universidades que promovieron la contratación inicial. El 15\% de los encuestados había cambiado su lugar de residencia dentro del país y, de estos, el 55\% lo había hecho para mejorar sus opciones laborales en educación superior.

(...) Nosotros vivíamos antes en Jipijapa... pero la universidad fue intervenida y nos echaron a todos. Como Luis había venido aquí, nos dijo que vinieramos a probar y hablar con la universidad, y hemos hablado ayer y nos han dado varias clases en economía. Para nosotros está muy bien, trabajo a media jornada, porque queremos hacer un doctorado y de otra manera, se junta todo y es imposible... se está bien aquí en Cuenca. (Diego, profesor universitario, Cuenca. 2014)

Según datos de la SENESCYT, la distribución de profesionales españoles a nivel nacional es bastante desigual, concentrándose en las regiones de Pichincha y en menor medida en Manabí, Guayas, Esmeraldas y Loja. Algo similar sucede con la distribución nacional que hasta el 2014 tenían los beneficiarios del programa Prometeo, ubicados primordialmente en Pichincha (35\%).

Con respecto a los ecuatorianos que retornan o aspiran a retornar para emplearse en educación superior se revelan datos significativos, como el número de ganadores del programa Prometeo (75 personas, es decir, el 12,6\%) o las postulaciones a la UNAE, que según el Ministerio de Educación, representan el 11,76\%, el segundo grupo nacional tras el español $(59,41 \%)$.

Por otra parte, los perfiles de los ecuatorianos en proceso formativo en España presentan diferencias notables. Encontramos primeramente un grupo que viaja de forma planificada y financiada desde Ecuador con ayuda del gobierno u otras entidades; se trata de sectores de clase media y media alta con un acceso más sostenido al sistema educativo y, en el caso de créditos, con garantes del pago. En segundo lugar, encontramos jóvenes ecuatorianos, hijos de migrantes residentes en España, muy afectados por la crisis y el alza de las tasas de la universidad española. Algunos optan por retornar o migrar a Ecuador para estudiar, mientras que otros se quedan postulando a las becas ecuatorianas.

Le propuse a mi padre irme a Ecuador a estudiar medicina. (...) A mí me apetecía un montón estudiar medicina. Claro mi padre 
me dijo que sí, que me apoyaba. Pero al final entre que se quedó en paro, no teníamos ingresos ninguno, entonces, si me iba Ecuador no iba a contar con ellos. Y empezar una nueva vida... aquí por lo menos tenía yo mi trabajo de fines de semanas que ese es mi sustento, para la Universidad y para todo. Porque mis padres no pueden ayudar. Entonces tomé la decisión: ¡Dije, mira, si me voy a Ecuador no cuento con nada! ¡Voy a estar ahí con mi familia pero no voy a poder vivir!, ¡Tendría que buscarme todo, todo desde el principio! Entonces decidí coger el grado de relaciones laborales porque es el siguiente paso de mi carrera; y el siguiente sería ya el master de prevención de riesgos laborales. Entonces ya culminaría con el master. Ya sería mi máximo objetivo a cumplir... Estoy en tercer año; me queda un año. (Lenin, estudiante universitario, 25 años, Madrid, 2014).

Si bien antes de 2008 se documentaban casos de jóvenes que habían migrado a España en la adolescencia y que decidían dejar los estudios (fundamentalmente secundarios) para insertarse tempranamente en el mercado laboral, ahora estos jóvenes vuelven a estudiar siguiendo trayectorias complejas. Es decir, dado que no han finalizado la secundaria en su momento, ingresan en formaciones profesionales o estudios de grado medio que les permiten acceder, en una segunda fase, a la universidad a una edad mucho más tardía. La trayectoria de sus hermanos y hermanas menores, nacidos en España o migrados entre 0 y 5 años, es más directa en el acceso (secundaria, selectividad, universidad), asemejándose a la de los autóctonos. No obstante, carecemos de datos sobre el acceso actual de esta población a la universidad, aunque de la investigación se deduce que va en aumento apoyándose en el ahorro familiar.

Su decisión de estudiar una carrera no está exenta de dificultades. Los recortes en educación han repercutido en las becas que servían para cubrir los gastos de matrícula, libros y transporte, además de elevarse los baremos para su otorgamiento. Estos jóvenes se han visto particularmente afectados por los recortes y muchos deben compatibilizar los estudios con un trabajo en la economía informal o en sectores precarios y mal pagados, lo cual alarga los periodos de estudio.

La mayor parte de los entrevistados opina que no tienen mucho futuro en España y plantean el retorno a Ecuador como una salida viable, aun cuando muchos apenas si han tenido contacto con el país. Como segunda posibilidad se plantean migrar a otros países 
europeos o a EEUU. Opinan que Ecuador es un país emergente «que necesita de sus aportes y conocimientos» y que ellos y ellas «quieren contribuir al desarrollo» de lo que consideran "su país». Es pronto para saber cuál será el destino educativo de estos jóvenes, cuyo acceso a la universidad recién comenzaba a hacerse efectivo. En todo caso, sus opciones habrán de estimarse junto a las de sus familias (Vega, 2014; Vega, Gómez y Monteros, 2015).

En definitiva, Ecuador se revela como un país de inserción laboral y no formativa para los españoles y, a la inversa, España sigue siendo un país para obtener títulos sin ninguna expectativa laboral para docentes y estudiantes ecuatorianos. Respecto a los ecuatorianos en universidades españolas se producen movimientos contradictorios: un incentivo a la circulación a través de la política educativa de becas y un incipiente y aún incierto retorno formativo para los afectados por la crisis.

\section{Condiciones de llegada y modalidades de inserción de españoles y ecuatorianos en educación superior}

Al valorar las condiciones laborales de la migración española, comparadas con las de otras poblaciones en el país (colombianos, haitianos, cubanos, etc.,), podemos concluir que son muy favorables (Zepeda y Verdesoto, 2011; Ortega y Ospina, 2012; Correa, 2014). El $80 \%$ de los encuestados se siente "satisfecho con su trabajo», y el $83 \%$ califica su vida en Ecuador «de buena», si bien refieren problemas administrativos (59\%) y retraso en la remuneración $(73,2 \%)$, cuestión que ha afectado particularmente a los beneficiarios del Prometeo. Destacan el acceso y las condiciones en el empleo como elementos positivos.

Pues mira, el tema de las condiciones laborales, pues la verdad es que me siento muy bien, tanto a nivel de salario... pues es un salario que está, la verdad, bastante por encima de lo que puedes cobrar allí... o lo que he cobrado yo, o lo que cobra cualquier profesor universitario allí, incluso siendo funcionario. (Julián, investigador prometeo, Quito, 2014).

Esta valoración se advierte también entre quienes regresan después de haber disfrutado de una beca del gobierno, si bien 
advierten el malestar de otros compatriotas que «se sienten un poco perjudicados».

... yo creo que las condiciones laborales para mí aquí son las mejores. Yo tengo la posibilidad y ahora la universidad está... ¡trabajando bastante bien! Y los sueldos que antes eran muy malos ahora son bastante buenos, para el país ¿no? Tener una cátedra allá sería imposible ¡con las condiciones que hay ahora! (...) (Soledad, docente e investigadora becada por SENESCYT, Cuenca, 2014).

Atendiendo a las dimensiones transnacionales de la inserción, la investigación permitió identificar tres modalidades que inciden en las condiciones laborales posteriores: una gestionada "desde arriba», es decir, vinculada al marco formal de las políticas educativas ecuatorianas; una desarrollada "desde abajo», que se apoya en redes no institucionales de colaboración; y finalmente, un nivel «intermedio», con una mediación para-estatal, como es el caso de la gestión de llamamientos a través de intermediarios (Guarnizo et al., 2003; Mahler y Hansing, 2005). En este último caso, hablamos de estrategias que articulan prácticas agenciales transnacionales entre lo macro y lo micro, lo local y lo global.

Ante las exigencias de las reformas ecuatorianas, las universidades han buscado mecanismos alternativos de llamamiento y selección de profesionales extranjeros, principalmente en España, imitando la gestión estatal. Esto explica la diversidad de actores involucrados y la variedad de trayectorias seguidas. En este sentido, la encuesta arrojó que el 3,1\% de profesionales españoles había llegado por ofrecimiento de instituciones españolas, frente al 67.5\%, que lo hizo por medio de instituciones ecuatorianas. El 19.4\% llegó a través de intermediarios, y un 10\% indicó otras vías.

Durante el trabajo de campo conocimos la existencia de un contacto ecuatoriano en España que realizaba funciones de captación y selección de españoles con el nivel demandado por las universidades ecuatorianas $^{30}$, en una suerte de «coyoterismo invertido» de migrantes

30 En uno de los ofrecimientos en redes sociales coordinado por esta persona, se puede leer: «Se necesitan 120 doctores en diferentes áreas, para trabajar como profesor y llevar a cabo labores de investigación en las distintas facultades que conforman dichas Universidades. El único requisito que se pide es ser doctorado en cualquier área y estar dispuesto a trasladarse y vivir allí un mínimo de dos años. A cambio se ofrece: 1.700 dólares/mes durante el primer año pudiendo llegar a duplicar la cuantía durante el segundo año. Todas las prestaciones sociales, 
cualificados alentado por las nuevas exigencias educativas. Este intermediario explica su labor del siguiente modo:

Con la mayor parte de las universidades de primer nivel, por ejemplo la Complutense, Politécnica de Madrid, la Autónoma, la Rey Juan Carlos, la Carlos III, la Universidad de Granada, Politécnica de Valencia, Universidad Pública de Navarra, con la Universidad de Valladolid, con muchas universidades. Y últimamente, para dar cumplimiento a la Ley Orgánica de Educación Superior, pues estamos contratando los PhDs. Yo me encargo del reclutamiento y de la selección de los PhDs, y de traerlos aquí a las universidades con las que trabajo. (...).

El proceso es simplemente que a través de las universidades con las que tenemos contacto, pues yo hago las convocatorias abiertas. Y se presentan los currículo, entonces hago todo un proceso técnico de selección, desde el análisis de los requisitos mínimos, y luego tengo entrevistas con cada uno de los candidatos. Primero individual y luego de manera colectiva. Creamos las condiciones para que se haga una selección, teniendo en cuenta no competencias cognitivas sino otro tipo de cosas, por ejemplo, la adaptación a un medio totalmente distinto. (...).

Yo, con las universidades con las que trabajo, a las que represento, hacemos una convocatoria pública en la página web de cada universidad, con las áreas de conocimiento, con las condiciones que les ofrecemos a nombre de las universidades ecuatorianas. Y las universidades me nombran a mí. Tengo una carta de nombramiento, en la que dicen que yo soy el responsable de la selección de estas personas. Si no, puede, aparentemente, puede parecer que es... (dice sonriendo) es un mediador o una trata de personas... no sé, un comisionista, esas cosas. ¡No! ¡Es parte de mi trabajo!

Como ilustra este caso, los actores y estrategias en universidades de menor reconocimiento se han ido diversificando, lo que implica, más allá de la lógica binacional y/o estatal, la articulación de actores públicos y privados en contextos de circulación transnacional. Si bien en algunos casos estas intermediaciones son castigadas como

cotizaciones a Seguridad Social, etc. Dos pagas extraordinarias al año. 30 días de vacaciones al año. 1 billete al año de avión, ida y vuelta a España. Las propias universidades ponen a disposición viviendas seleccionadas y a muy buen precio para que elijan los candidatos seleccionados». Tomado del Blog Juventud de la Federación de Trabajadores de la Enseñanza, http://feteugtmadrid.wordpress. com/2012/10/09/oferta-de-empleo-para-doctorados/ 
ilegales o a-legales, cuando se trata de la movilidad de cualificados, especialmente en desplazamientos Norte-Sur, lo que advertimos es una consideración totalmente distinta. Frente a la migración no cualificada, comprendida en términos de lastre económico (Delgado et al., 2009), la de titulados es estimulada acudiendo a mecanismos formales, pero también a otros menos formales.

Esta movilidad también se produce a nivel de las redes de amistad y familia. La proyección de estabilidad u oportunidad de uno de los miembros de la familia en destino puede desencadenar un proceso de inserción paralelo. Si tenemos en cuenta el habitual emparejamiento entre académicos, esto resulta bastante común.

Surgió la oportunidad que Marta encontró un trabajo (en Ecuador) porque le dijeron unas amigas y entonces lo consiguió, y ya era como algo fijo, para establecernos y detrás vine yo. Yo soy economista y tengo un máster hecho en Comercio exterior [...] De hecho, en España no he trabajado, apenas unas prácticas que hice recién terminada la universidad. Y, de hecho, no he vuelto a trabajar en España, siempre he estado en Londres, vaya. (Roberto, profesor universitario, Cuenca, 2014).

En cuanto a las condiciones de los ecuatorianos en universidades españolas, además de las diferencias ya señaladas entre becados desplazados e hijos de ecuatorianos residentes, cabe resaltar que la naturaleza no reembolsable de las ayudas hace que esta opción sea muy beneficiosa, aunque se presente condicionada por la obligación de regresar. La formación se dirige fundamentalmente a áreas técnicas estratégicas para el cambio de la matriz productiva.

En cuanto a los jóvenes ecuatorianos universitarios residentes en España que deciden retorar, sus redes de contacto e información se están desarrollando fundamentalmente "desde abajo», a través de familiares y, más recientemente, de compañeros de estudios o amigos. El optimismo en conseguir empleo cualificado en Ecuador es manifiesto mientras todavía continúan en España. Existe una modalidad novedosa de red migratoria de estudiantes ecuatorianos conectada con la organización política: jóvenes con inquietudes que constituyen asociaciones en las que comparten problemas, experiencias e intercambiar información y apoyos con vistas al retorno. Estas redes, más articuladas, evidencian los procesos transnacionales que intervienen en la circulación de los migrantes al poner en contacto a jóvenes en ambos países. 


\section{CONCLUSIONES}

En la actualidad, los destinos de españoles y ecuatorianos en los sistemas educativos de ambos países se hallan interconectados en un espacio, la educación superior, que se erige en torno a lógicas interdependientes de movilidad circular. Dicha conexión viene determinada por el contexto crisis y oportunidad, así como por el diferencial de valor y de acceso a la educación en uno y otro país en relación a otros posibles destinos.

La declaración de las autoridades ecuatorianas de querer elevar el nivel educativo en relación a las universidades de prestigio internacional sin mermar el acceso de sectores populares genera no pocas contradicciones y polémicas en un entorno en el que se alude a la soberanía y la reubicación del Ecuador en la economía global del conocimiento (Villavicencio, 2014). Con frecuencia, las desigualdades globales, pero también nacionales, quedan soslayadas, al igual que los criterios de «excelencia» que dominan la internacionalización de la educación superior. En este escenario, la disputa sobre las valoraciones y significados que rodean la educación en relación a la movilidad adquiere una relevancia crucial que ha de ser estimada a la luz de las estrategias de actores e instituciones.

La inserción laboral y formativa de personas ecuatorianas y españolas en ambos países aparece dinámicamente entrelazada. La atracción que ejercen las políticas educativas ecuatorianas, especialmente hacia aquellos sectores que han podido acumular cualificaciones reconocidas y de prestigio, ha generado desplazamientos encadenados: de españoles para insertarse en las universidades ecuatorianas, pero también de ecuatorianos para obtener títulos de postgrado en el extranjero con los que garantizar futuros empleos. El retorno de éstos últimos y de otros ecuatorianos y la llegada de españoles en los próximos años dependerá, al menos parcialmente, de las opciones de estabilidad o nuevas posibilidades de movilidad de los primeros en relación a sus carreras y estrategias de vida.

Si bien las políticas educativas ecuatorianas parecen impulsar la consolidación de plantas docentes en distintos niveles del escalafón, limitando la contratación temporal de «externos», las anunciadas reformas laborales en Ecuador, con la eliminación de contratos fijos, caminan en un sentido inverso. Esto podría significar una polarización entre un cuerpo creciente de titulares indefinidos y 
un contingente aún incierto de profesorado «flotante» sin relación de dependencia, entre los que podrían situarse los migrantes y retornados de la crisis que están llegando a Ecuador en estos momentos, así como los futuros titulados con obligación de regresar pero con trayectorias profesionales aún no consolidadas. Sin duda, esto podría implicar una redirección en los flujos de salida desde España.

Por otra parte, la creciente degradación de la educación en España, junto con un encarecimiento de las matrículas, podría implicar en poco tiempo una pérdida de prestigio, y potenciales desplazamientos de estudiantes nacionales y extranjeros, con recursos, becas o familias en origen, hacia el exterior. La capacidad de las universidades españolas para captar la matrícula de extranjeros con becas externas tendrá que considerarse en relación a las oportunidades que brinden otros destinos.

En estos momentos, en los que la cooperación al desarrollo ha dejado de ser una opción para los españoles en Ecuador, la educación se perfila como un campo de inserción atractivo y prestigioso para los que llegan, incluyendo a los propios ecuatorianos cualificados. No obstante, como demuestra nuestra investigación, el destino de una parte de los prometeos que finalizan sus contratos, de los que llegan en migraciones y retornos cada vez menos planificados, pero incluso de aquellos que ha estabilizado su situación, resulta aún incierta, como bien lo ponen de relieve sus testimonios.

\section{BIBLIOGRAFÍA}

Alaminos, A., Sancreu, O. y Albert, M. (2010): «La movilidad social de inmigrantes españoles en Europa». REIS, 129, pp. 13-35.

ARAB, Ch. (2008): «La circulation migratoire: Une notion pour penser les migrations internationales». En e-migrinter, No. 1, disponible en: http:// www.mshs.univ-poitiers.fr/migrinter/e-migrinter/200801/emigrin ter2008_01_20.pdf

CACHÓN, L. (2012): «La inmigración de mañana en la España de la Gran Recesión y después». Panorama Social, 16, pp. 71-83.

Correa, A. (2014): Del Caribe a la Mitad del Mundo. Migración cubana en Ecuador. Quito, Abya-Yala.

Delgado, R., Márouez, H. y Rodríguez, H. (2009): «Seis tesis para desmitificar el nexo entre migración y desarrollo». En Migración y Desarrollo. 
Zacatecas. No. 12. Disponible en: http://www.scielo.org.mx/scielo. php?script=sci_arttext\&pid=S1870-75992009000100002

Docouiere,F.yRapoport,H.(2006): «TheBraindrain».Disponibleen:http:/www. researchgate.net/profile/Frederic_Docquier/publication/228394436_ The_brain_drain/links/0912f50c8bb85b0615000000.pdf

GIL, S. (2006): "Las argucias de la integración. Construcción nacional y gobierno de lo social a través de las políticas de integración de inmigrantes. Los casos de Cataluña y Madrid». Tesis doctoral. Universidad Complutense de Madrid.

Glick Schiller, N., Basch, L. y Szanton Blanc, C. (1999): «From Immigrant to Transmigrant: Theorizing Transnational Migration». En PRIES, L. (ed.) Migration and Transnational Social Spaces. Farnham, Ashgate, pp.73-105.

GonzÁlez, C. (2013): «¿Emigran los españoles?». Real Instituto Elcano, Demografía, Población y Migraciones Internacionales.

- (2015): «Guerra de cifras: los jóvenes emigrados». Disponible en: http:// www.blog.rielcano.org/guerra-de-cifras-los-jovenes-emigrados/

GonzÁLEz-Ferrer, A. (2013): La nueva emigración española, lo que sabemos y lo que no. Madrid, ZOOM Político, KIEGD-CSIC.

Gómez, S. y de la CALle, A. (1995): Las relaciones laborales en España. Ed. Cátedra SEAT de Relaciones Laborales-Universidad de Navarra. Barcelona.

Guarnizo, L. E., Portes, A. y Haller, W. J. (2003): «Assimilation and Transnationalism: Determinants of Transnational Political Action among Contemporary Migrants». American Journal of Sociology, 108, 6, pp. 1211-1248.

Herrera, G., Moncayo, M., Escobar, A. (2012): Perfil migratorio del Ecuador 2011. Ecuador, Organización Internacional para las Migraciones (OIM), Ecuador.

Herrera, G. (2007): «Ecuatorianos en Europa. De la salida vertiginosa a la construcción de espacios transnacionales». En Yepez, I. y Herrera, G. (eds.), Nuevas migraciones latinoamericanas a Europa. Balances y desafíos. Quito, FLACSO-GRIAL-OBREAL, pp. 189-215.

Hily, M. A. y Ma Mung, U. (2003): «Catégories et lieux des circulations migratoires». Cahiers de Recherches de la Mire, pp.33-39.

Hochschild, A. (2001): «Las cadenas mundiales de afecto y asistencia y la plusvalía emocional». En Giddens, A. y W. Hutton, (eds.). La vida en el capitalismo global. Barcelona, Kriterios Tusquets, pp. 187-208.

JoKish, B. y KYLE, D. (2005): «Las transformaciones de la migración transnacional del Ecuador, 1993-2003». En Herrera, G. et al. (eds.). La migración ecuatoriana transnacionalismo, redes e identidades, Quito, FLACSO, pp. 57-70.

LóPEz, I. y RoDRíGuEz, E. (2010): Financiarización, territorio y sociedad de propietarios en la onda larga del capitalismo hispano (1959-2010). Madrid, Traficantes de Sueños. 
Ma Mung, E., et al. (1998): "La circulation migratoire, bilan des travaux. Synthèse». Migrations études, ADRI (Agence pour le développement des relations interculturelles), pp.1-12.

Mahler, J. y Hansing, K. (2005): Toward a transnationalism of the middle. How transnational religious practices help bridge the divides between Cuba and Miami. Latin American Perspective, Issue 140, Vol. 32 No. 1, January.

Moncayo, I. (2011): Migración y retorno en el Ecuador. Entre el discurso político y la política de gobierno. (Tesis). Quito, FLACSO-Ecuador.

Navarro, V., Torres, J. y Garzón, A. (2011): Hay alternativas. Propuestas para crear empleo y bienestar social en España. Madrid, Sequitur \& Attac España.

Ortega, C. y Ospina, O. (coord.). (2012): No se puede ser refugiado toda la vida. Refugiados urbanos: el caso de la población colombiana en Quito y Guayaquil. Quito: FLACSO, Sede Ecuador. 261 p.

Oteiza, E. (1971): «Emigración de profesionales, técnicos y obreros calificados argentinos a los Estados Unidos: análisis de las fluctuaciones de la emigración bruta, julio 1950 a junio 1970», Desarrollo Económico. Vol. 10, No 39/40, pp. 429-454.

Pedone, C. (2005): «Tú siempre jalas a los tuyos. Cadenas y redes migratorias de las familias ecuatorianas hacia España». En Herrera, G., Carrillo, M.C. y Torres, A. (eds.) La migración ecuatoriana transnacionalismo, redes e identidades, Quito, FLACSO, pp.105-143

Polavieja, J. G. (2006): "The Incidence of Temporary Employment in Advanced Economies: Why Is Spain Different?»European Sociological Review, 22/1, pp. 61-78.

Prats, J. (2005): «El sistema educativo español». En Raventós, F. y J. Prats (dirs). Los sistemas educativos europeos ¿Crisis o transformación? Barcelona, Editorial La Caixa. Colección Estudios Sociales. pp. 177-228.

RAMíREZ, R. (2010): Transformar la Universidad para Transformar la Sociedad. Quito, Ed. SENPLADES.

Sallé, M. A. (2009): La migración española en América: historia y lecciones para el futuro, Madrid, Fundación directa.

SANZ ABAD, J. y GARcía, C. (2012): La pluralidad y heterogeneidad de las migraciones internacionales: proyectos migratorios por razones extraeconómicas. Aportaciones a partir del estudio de mujeres cubanas y ecuatorianas en España», VII Congreso Migraciones Internacionales en España, Bilbao, abril 2012.

VEGA, C. (en prensa): «El retorno más allá del mito del emprendedor. Estrategias económicas, familiares y afectivas de mujeres y hombres a su regreso a Ecuador desde España», Papers.

Vega, C., Gómez, C. y Monteros, S. (2016): Jóvenes ecuatorianos, movilidad y educación superior en España y Ecuador. Desigualdad social y trayectorias educativas en tiempos de crisis. Ponencia en XVI congreso de migración: Migraciones y Estrategias frente a la crisis, CEMYRI, Universidad de Almería, 10-12 de marzo 2016. 
Sevilla, C. (2010): La fábrica del conocimiento. La universidad-empresa en la producción flexible. Madrid, El Viejo Topo.

Sheller, M. y Urry, J. (2014): «El nuevo paradigma de las movilidades». En Ribas-Mateos, N. y Laiz, S. (eds) Movilidades adolescentes. Elementos teóricos emergentes en la ruta entre Marruecos y Europa, Barcelona, Ediciones Bellaterrra, pp. 63-101.

Villavicencio, A. (2014): «Universidad, conocimiento y economía», Pre-textos para el debate, 1, Quito: Universidad Andina Simón Bolivar.

TARRIUS, A. (2000): «Leer, describir, interpretar las circulaciones migratorias: conveniencia de la noción de territorio circulatorio. Los nuevos hábitos de la identidad». Relaciones. Estudios de historia y sociedad, XXI, 83, El Colegio de Michoacán, A.C México, pp. 38-66.

Zepeda, B. y Verdesoto, L. (2011): Ecuador, las Américas y el mundo 2010. Opinión pública y política exterior. Quito, Flacso-Ecuador. 